\title{
Musings on Hardware Advances and New Directions
}

Eiichi Fukushima

\section{1}

\section{Scope and Introduction}

This chapter is an edited version of a rambling tutorial talk given at the 9th ICMRM in 2007, followed by a longer discussion of modern permanent magnets being used for NMR. All of this is supposed to be couched in 'nuts and bolts' terms, but that is a phrase which is often applied to my presentations regardless of the subject-it should not be taken too literally.

NMR/MRI is an extremely broad field, both in terms of the physics that is the basis for the field as well as its applications. In these senses, it is truly unique among all techniques. It was the aim of the tutorial lectures to provide sufficient background for a better understanding of the varied presentations given at the conference.

One dominant discipline that uses NMR is NMR spectroscopy for analytical chemistry and biochemistry. This was made possible when chemical shift was discovered shortly after the inception of the field. MRI, which can be thought of as spatially resolved NMR as opposed to chemically resolved NMR of NMR spectroscopy, is a relative latecomer to the field; it has been around for roughly $50 \%$ of the approximately 60 -year history of NMR. Then, there is the original 'physically resolved' NMR, where the contrast mechanism consists of any number of relaxation parameters that are affected by molecular structure and motion. Thus, the parameter space for NMR/MRI can be thought of as shown in Figure 1.1. The three regions are the three overall contrast mechanisms (chemical, physical, spatial), and the intersections of the regions contain some of the most interesting applications. For this audience, they include velocity and diffusion imaging (as described by some of the chapters in the Transport section of this book), NMR well-logging (as described by Lizhi Xiao in Chapter 31), and NMR elastography. 


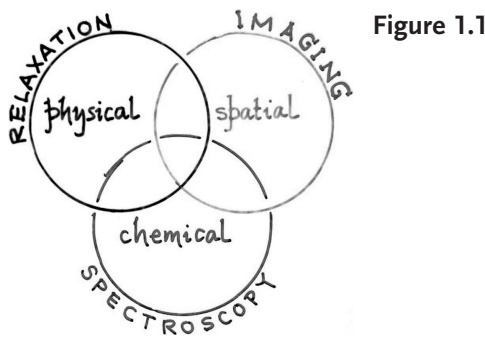

\section{2}

\section{NMR Building Blocks}

NMR experiments have broad analogy to radio/television broadcast and reception. As shown in Figure 1.2, the objective of radio/TV broadcast and reception is to receive what was broadcast with as few changes as possible. However, if there is any change in the quality of reception, it is possible to characterize the medium that changed the signal if the input and output (i.e. what was transmitted and received) are known. In the same way, NMR starts with a known transmitted signal-for example, a rectangular pulse or a sinc pulse-and analyzes the sample (the intervening medium) by deconvoluting the received signal by the transmitted signal.

Thus, the NMR/MRI apparatus consists broadly of source of radiofrequency (RF) excitation, an NMR probe that interacts with the sample, a signal receiver, a

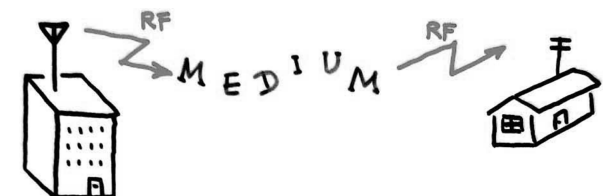

Transmitter

Receiver

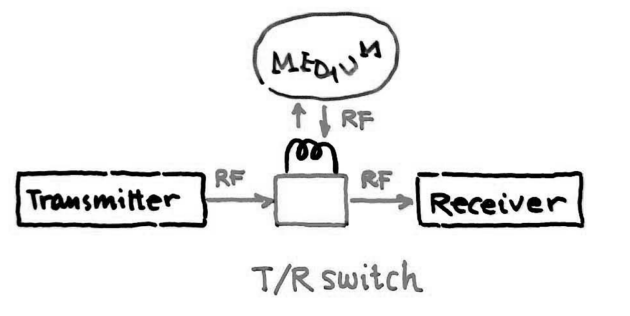

$R F \equiv$ radio frequency magnetic field.

Figure 1.2 


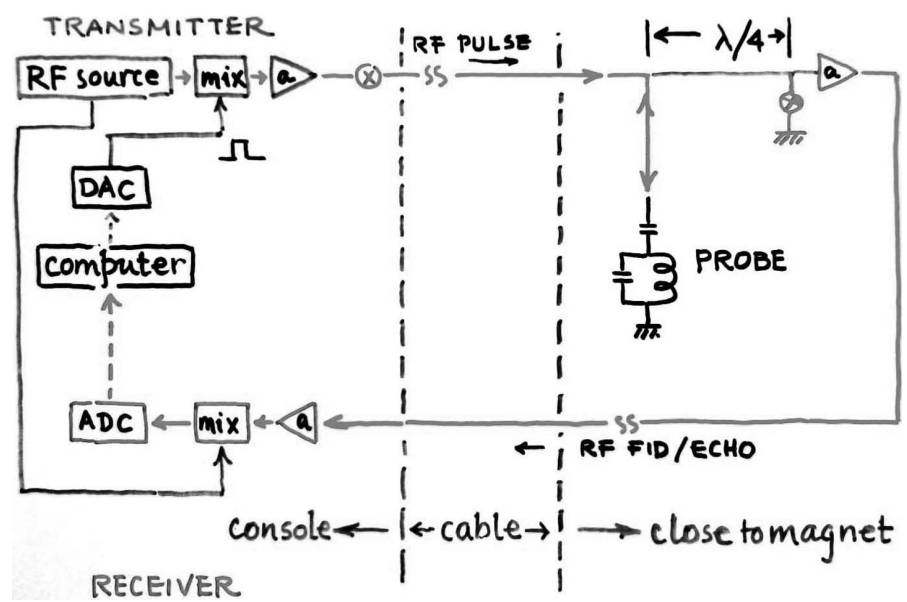

Figure 1.3

source of the magnetic field, and a controller that keeps order. Figure 1.3 is a block diagram of a typical NMR apparatus. Some common requirements are dictated by the typical signal size of few to tens of microvolts that needs to be amplified 100000 to 1000000 -fold in order to be detectable.

Many of the requirements vary over many orders, depending on the application. The magnetic field strength can vary from the Earth's field (e.g. Halse, et al. in Chapter 2) to few tens of Tesla, a range of five orders of magnitude. (Some of the strongest field magnetic resonance experiments have been carried out, not in magnets but in the vicinity of strongly polarized atoms.)

One important parameter is the recovery time of the NMR apparatus after an excitation, as this determines how soon the signal can be seen. The difficulty of obtaining short recovery times is the main reason for the relative slowness in the progress of imaging objects with short $T_{2}$. The common MRI sequences that use spin echoes rely on the samples having a long spin-spin relaxation time $T_{2}$, so they do not rely on short-recovery-time hardware. Thus, they are most suitable for low-viscosity liquids - which happen to be abundant in many systems, especially in biology. The question of how to study short(er) $T_{2}$ samples was the subject of several presentations at this conference.

\section{3}

\section{NMR of Short- $T_{2}$ Samples}

Liquids tend to have $T_{2} \sim T_{1}$ while solids tend to have $T_{2} \ll T_{1}$. Therefore, it is much easier to obtain big signals from liquids than solids (because the short $T_{2}$ may make the signal decay in time short compared to the recovery time of the NMR apparatus). The general rule is that the more something is like a solid, the shorter $T_{2}$ becomes. This means normal tissues might have a quite long $T_{2}$, whereas 
cartilages, bones and lungs have very short $T_{2}$ and are not commonly imaged in clinical MRI units. Today, hardware and pulse sequence improvements are available that address this issue, and some of the presentations at the conference have dealt with this problem. Thus, there will be an increasing number of NMR (and imaging) of shorter $T_{2}$ samples such as soft bones, lungs, plastics, and so on, performed in the near future as we improve our ability to measure short- $T_{2}$ samples easily, and especially for larger samples.

\section{4}

\section{Field Dependence of Signal Strength}

The signal-to-noise ratio (SNR) of NMR signals depends strongly on the strength of the static magnetic field. Conventional wisdom states that the SNR goes up as frequency to the $7 / 4$ th power. This fact is the driving force behind the push to perform experiments at ever stronger magnetic fields. (It might be interesting to know that it was believed early in MRI that whole-body imaging in fields higher than $1 \mathrm{~T}$ would not be possible because of electromagnetic absorption and other problems, although I have now heard of a $7 \mathrm{~T}$ whole-body scanner which should have a SNR that is enhanced from the original estimate by around 30fold.) Today, the instrument manufacturers now pushing to raise the field above $23 \mathrm{~T}$ for high-resolution NMR which means a proton resonance frequency of $1 \mathrm{GHz}$

So, how is it possible for some of us, especially at this conference, to consider performing NMR (and MRI) at significantly weaker fields? One helping factor is that it is usually possible to use solenoid coils at the weaker fields because the magnet geometry is different at the lower fields than at the axial fields of normal, superconducting magnets. Solenoids are better coils than the normal type used in common cylindrical superconducting magnets with an axial field (e.g. birdcage, saddle and Alderman-Grant coils) because the effective turns density is higher. Thus, the penalty of moving to weaker fields is partly offset by the availability of solenoid coils.

Another approach is simply to make the sample larger to compensate for the loss of signal at the weaker field, and this is done in many geophysical NMR applications, including borehole logging.

\section{5}

\section{Sample Size Dependence}

NMR spans the range from the subsurface detection of water with $100 \mathrm{~m}$ surface coils to microcoil studies with samples that are fractions of a nanoliter. In units of milliliters, the sample for the surface coil is 10 or 11 orders of magnitude larger, while the microcoil samples are six orders or more smaller. This total range of 16 to 17 orders is difficult and unusual for any measuring method. 
Transmitter and Receiver Coils

Coils are the all-important link between the sample and the measuring apparatus-as is well-known, a bad coil can completely ruin an experiment. So, there is a choice of either using separate coils for transmitting and receiving, or the same coil can be multiplexed to perform both tasks. Separate coils are used often in clinical imagers where the transmitter coil is built into the magnet housing while the receiver coil is tailored to the sample. This is because a bad transmitter coil only increases the need for more input power, whereas a bad receiver coil will lead to loss in SNR that is not recoverable. Therefore, we can usually tolerate inefficiencies in transmission, but not in reception.

One criterion for a good (receiver) coil is that it generates an intense magnetic field at the sample per unit current in the coil. David Hoult proved this using the reciprocity principle [1]. This seemingly simple principle leads to some interesting conclusions that are not always appreciated. For a given overall coil geometry, the turns density is important because the field generated is directly proportional to the number of turns. For identical windings, the quality factor $Q=\omega L / r$ can be maximized to limit the bandwidth, although the bandwidth does not have to be set by the NMR coil. Andrew McDowell has a scheme to tune microcoils with terrible $Q$-values due to thin wires that have substantial resistivity [2] at frequencies that are too low to deal with convenient values of tuning capacitors. He obtains excellent SNRs by connecting the microcoils in series with large, high-Q dummy coils. The microcoil acts as a resistor to the tank circuit, while the dummy coil sets the resonance condition. Additional descriptions of these coils will be provided later, in conjunction with the accompanying ultracompact magnet.

\section{7}

\section{Shrinking Magnets}

Here again, I will discuss a 'fringe' area of NMR and MRI. The most common magnet in NMR/MRI is the persistent-mode superconducting magnet that generate fields along the axis, having replaced the iron core electromagnet as the dominant magnet in the $1970 \mathrm{~s}$, for good reasons. These magnets create very strong and uniform fields that are stable, but they are expensive to buy and neither cheap nor easy to maintain. This latter fact may be the single most important factor in these magnets not becoming the basis for common uses of NMR/MRI outside laboratories, for example in factories, stores and outdoors. However, this situation is slowly changing with the modern permanent magnet, which performs better than ever before, are easy to maintain, require small installation spaces, and are relatively inexpensive to purchase and to maintain. The re-emergence of these magnets is due, in part, to the discovery of new rare-earth-based magnetic materials such as $\mathrm{SmCo}$ and $\mathrm{NdFeB}$. However, another important reason is the realization that 
Table 1.1 The advantages and disadvantages of the various magnet types.

\begin{tabular}{lll}
\hline Type of magnet & Strengths & Weaknesses \\
\hline Permanent & $\begin{array}{l}\text { Small size; low maintenance; } \\
\text { cheapest }\end{array}$ & Weaker field \\
Electromagnet & Variable field strength & Weaker field \\
Superconducting & $\begin{array}{l}\text { Strong field; most stable field } \\
\text { strength }\end{array}$ & High maintenance; most expensive \\
\hline
\end{tabular}

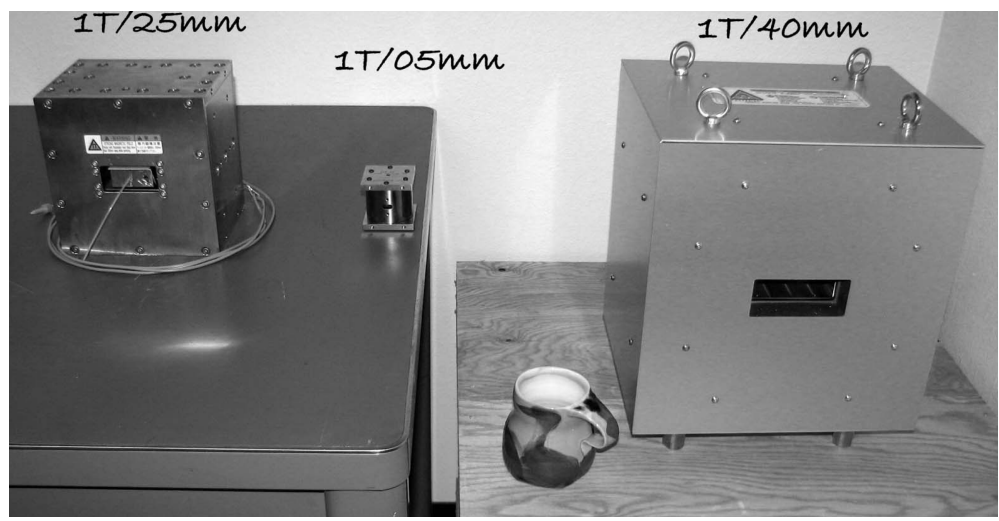

Figure 1.4

NMR can achieve much at the relatively low field, and this was not realized in the past.

In Table 1.1 the advantages and disadvantages are listed of a variety of magnets, including those electromagnets not mentioned previously. Although electromagnets are convenient for changing field strengths, they otherwise are bulky, expensive (due to the need for fairly elaborate power supplies), and they suffer from short-term field fluctuations.

The effect of the coil that offsets some of the loss in SNR in going from a highfield superconducting magnet to a lower-field permanent magnet was mentioned previously. Therefore, these permanent magnets-which are relatively compact, inexpensive and maintenance free-will become increasingly popular. Figure 1.4 shows several small permanent $1 \mathrm{~T}$ magnets (plus a coffee cup for scale comparison) that are used in our laboratory. The two larger magnets (manufactured by NEOMAX, a division of Hitachi Metals Co. in Japan) are NdFeB magnets with gaps of 40 and $25 \mathrm{~mm}$, and have homogeneity of $10 \mathrm{ppm}$ over half of the gap, without shim coils. The smallest unit is a SmCo magnet (made by Aster) which 


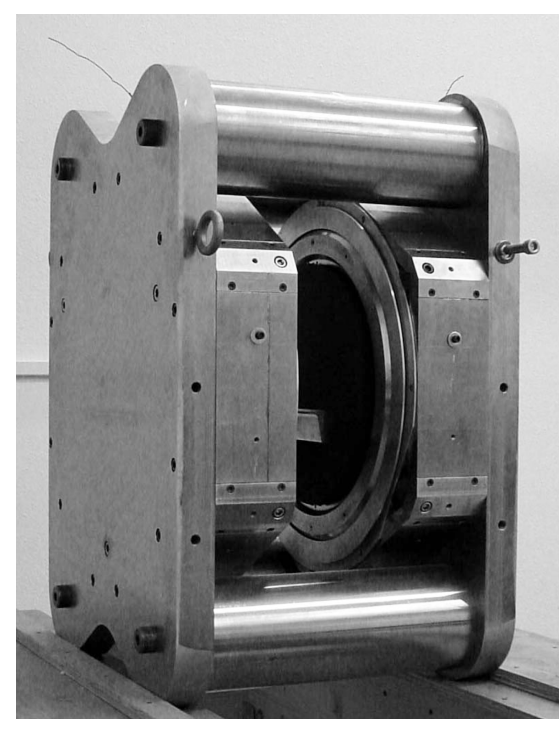

Figure 1.5

has a gap of $5 \mathrm{~mm}$, weighs $700 \mathrm{~g}$, and has an unshimmed homogeneity of $1 / 4 \mathrm{ppm}$ with a $300 \mu \mathrm{m}$ microcoil sample. The NdFeB material is inexpensive and strongly magnetized, but has the disadvantage for NMR of a large temperature coefficient of the field $\left(\sim-1000 \mathrm{ppm} \mathrm{K}^{-1}\right)$. Therefore, temperature regulation and a field-frequency lock are advisable. In order to take advantage of $1 / 4 \mathrm{ppm}$ homogeneity, the temperature would have to be regulated to within 250 microdegrees if there were no field-frequency lock present. Unfortunately, this is a daunting task and will limit sample access because of the significant insulation that would be needed. Thus, it makes sense to divide the task of field stability between temperature regulation and field-frequency lock.

For larger gaps, the magnet in Figure 1.5 should be considered. This $0.27 \mathrm{~T} / 150 \mathrm{~mm}$ magnet, which is produced by Aster, is big and heavy and has vertical and horizontal access, which is a good feature to have for flow NMR. There is no fringe field due to the substantial return paths provided by the four cylindrical posts, and the gradient coils take up almost $25 \mathrm{~mm}$, so that the space available for the probe is approximately $125 \mathrm{~mm}$.

The NEOMAX magnets such as those shown in Figure 1.4 are also available in larger sizes. For example, the $1 \mathrm{~T} / 60 \mathrm{~mm}$ magnet which we have used in our laboratory for a number of years weighs approximately $220 \mathrm{~kg}$ and can easily be moved around on a wheeled stand. Although it has been used for mouse imaging, the small gap (the $60 \mathrm{~mm}$ measurement does not include the gradient coils) limits its usefulness when compared to more modern NEOMAX magnets that leave a $60 \mathrm{~mm}$ gap, even with the gradient coils installed. The newer magnets also have crossed access slots at right-angles so that it is possible to have both vertical and horizontal access at the same time. 
The largest magnet in this family is a modern $1 \mathrm{~T} / 100 \mathrm{~mm}$ magnet described by Kose in Chapter 23 (see Figure 23.6). This magnet has a footprint of approximately $1 \mathrm{~m}^{2}$, and the entire NMR/MRI system can be fitted into a space twice that size. The magnet's homogeneity can be shimmed to $16.4 \mathrm{ppm}$ over a $60 \mathrm{~mm}$ diameter spherical volume, while the magnet temperature can be regulated at around $30^{\circ} \mathrm{C}$ to minimize temperature drift of the Larmor frequency.

The NEOMAX magnets are what I refer to as 'modified' Halbach magnets. The standard Halbach magnets are annular arrangements of magnetic materials which generate transverse fields that are uniform, provided that the annulus is long compared to the diameter [3]. It is difficult to obtain superb uniformity with a standard Halbach magnet because the field homogeneity depends on the uniformity of the magnetic material, which is difficult to achieve at present. The NEOMAX magnets alleviate these difficulties by 'squaring' the geometry, thus enabling the use of steel pole caps to define the gap [4]. Now, the burden shifts to the accuracy of the pole caps' geometry plus balancing the flux on the two sides-which are much more manageable tasks. In addition, the magnetized blocks are arranged to shorten the magnet, resulting in an almost cubic aspect ratio.

\section{8}

\section{Shrinking NMR}

In several presentations at the conference the details of small instruments that, by necessity, used small magnets and miniaturized electronics, were discussed. In fact, a consortium has now been set up which is headed by Peter Blümler and called the Virtual Institute for Portable NMR (http://www.portable-nmr.eu/index. php?index=2). Several commercial efforts have also been undertaken to create a compact NMR, mainly because a significant proportion of the cost (and weight) of any NMR or MRI is due to the magnet, so the instrument would be both cheaper and lighter if the magnet were to be made very small. The fact that there may be a 100- to 1000-fold reduction in the cost of the magnet harbors great promise for the future of ultra-compact (even hand-held?) NMR/MRI.

Andrew McDowell has pioneered the use of microcoils (coils smaller than $500 \mu \mathrm{m}$ ) with permanent magnets $[2,5]$. As already mentioned, a major challenge here was in determining how to tune a coil with such a small inductance at low frequency, but this was overcome nicely with an auxiliary (normal size) coil that set the resonance condition with a normal tuning capacitor [2].

Figure 1.6 shows the first proton spectrum acquired using the smallest magnet in Figure 1.4. The drawn curve is a Lorentzian fit with width $9.9 \mathrm{~Hz}$. The magnet used weighs $685 \mathrm{~g}$, has a volume of $125 \mathrm{~cm}^{3}$, requires no adjustments, is nearly impervious to external magnetic fields, and responds gracefully to temperature variations - that is, the field profile does not change as the center value of the field changes. Most impressively, a microcoil probe built for this magnet achieves $0.25 \mathrm{ppm}$ resolution without the use of current shims from $21 \mathrm{nl}$ of water to provide a SNR of 61 in a single data acquisition. 


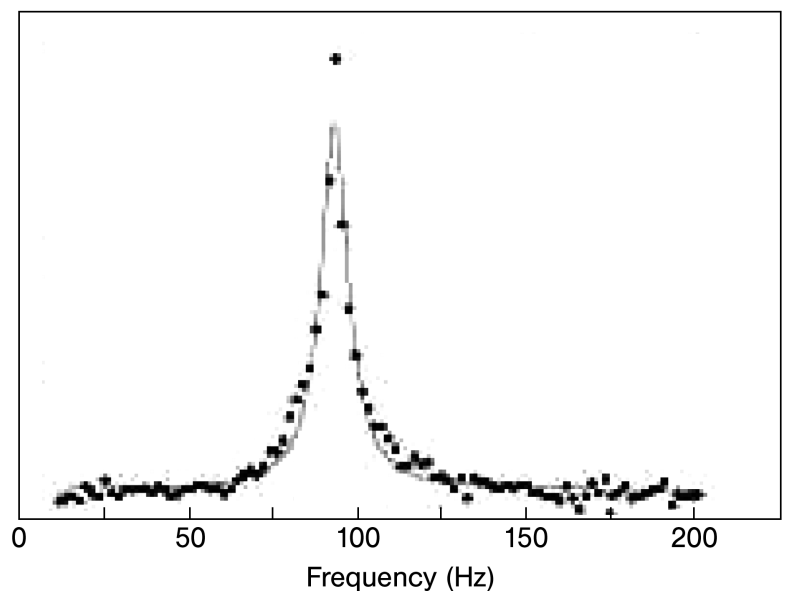

Figure 1.6

The eight-turn coil was hand-wound on a glass capillary with a $400 \mu \mathrm{m}$ outer diameter and $300 \mu \mathrm{m}$ inner diameter. Enameled magnet wire (50 gauge, total diameter $37 \mathrm{~mm}$ ) was used for the winding. To date, the diameters of the capillaries used have ranged from 100 to $550 \mu \mathrm{m}$. The $400 \mu \mathrm{m}$ size was chosen as a compromise between enclosing enough sample volume to provide adequate SNR performance and the need to probe the smallest practical region of the magnetic field in order to achieve a narrow line width.

When combined with existing single-board NMR spectrometer technology, such as a SpinCore board [6], this magnet and probe would be a hand-held, frequencydomain NMR spectrometer. The beauty of such a small NMR is that no power amplifier is needed, although the RF output of the transmitter may have to be attenuated to make this apparatus work.

Another area in which small and portable magnets will be used is unilateral NMR. This is a field that has been developed to a high degree of sophistication by the Aachen group in the form of NMR MOUSE [7]. A major application has been to the cultural heritage [8], and we at New Mexico Resonance and ABQMR have also been dabbling in this field. Our idea is to trade the high sensitivity of the MOUSE for a greater distance of detection with a barrel magnet [9]. Without going into details, this magnet is better at farther distances because it creates a 'sweet spot' where the field is reasonably uniform, thus enlarging the sensitive volume. Because of this uniform field spot, it should also perform well when measuring $T_{2}$ and the parameters derived from it, such as the diffusion constant. McDonald [10] and Marble [11] have presented different versions of such a unilateral magnet, while Callaghan's group has also developed a device that is closely related to ours [12].

Finally, the petroleum industry uses bore-hole well loggers which detect NMR signals from the material outside boreholes from tools within bore-holes, as 
described by Xiao [13]. However, even though these incredible devices are obviously mobile, they are neither small nor cheap.

\section{9}

\section{Future Prospects}

Who knows what the future holds? Nobody predicted many of the recent advances in this field, such as even chemical shift, NMR imaging or the Earth's field Jcoupling spectroscopy experiments [14]. Who would have thought NMR would be performed in the grasslands of Siberia [15] or on the Ross Ice Shelf in Antarctica [16]?

Having said that, there are certain points for the future which must surely come true. First, there will be a future! Besides the predictable directions of using higher fields and developing more sophisticated ways in which to manipulate spins, there will be diversification in new directions, as I have implied here. There will also be a spread of NMR/MRI techniques to areas that have not yet been exposed to such technology, or have been to only very limited degrees. This whole advance will be aided by the miniaturization of the magnets and electronics -it may not be too long before there is a cell-phone version of NMR!

\section{References}

1 Hoult, D.I. and Richards, R.E. (1976) The signal-to-noise ratio of the nuclear magnetic resonance experiment. Journal of Magnetic Resonance, 24, 71-85.

2 Sillerud, L.O., McDowell, A.F., Adolphi, N.L., Serda, R.E., Adams, D.P., Vasile, M.J. and Alam, T.M. (2006) 1H NMR detection of superparamagnetic nanoparticles at $1 \mathrm{~T}$ using a microcoil and novel tuning circuit. Journal of Magnetic Resonance, 181, 181-90.

3 Halbach, K. (1980) Design of permanent multipole magnets with oriented rare earth cobalt material. Nuclear Instruments and Methods, 169, 1-10.

4 Aoki, M. and Tsuzaki, T. (2006) Magnetic field generating device and MRI equipment using the device, U. S. Patent No. 7,084,633 (August 1).

5 McDowell, A.F. and Adolphi, N.L. (2007) Operating nanoliter scale NMR microcoils in a 1 Tesla field. Journal of Magnetic Resonance, 188, 74-82.

6 SpinCore Technologies, Inc. (http:// www.spincore.com).
7 Eidmann, G., Savelsberg, R., Blümler, P. and Blümich, B. (1996) The NMR MOUSE, a mobile universal surface explorer. Journal of Magnetic Resonance, A122, 104-9.

8 Proietti, N., Capitani, D., Rossi, E., Cozzolino, S. and Segre, A.L. (2007) Unilateral NMR study of a XVI century wall painted. Journal of Magnetic Resonance, 186, 311-18.

9 Fukushima, E. and Jackson, J.A. (2004) Unilateral magnet having a remote uniform field region for nuclear magnetic resonance, U.S. Patent No. 6,828,892 (December 7).

10 McDonald, P.J., Aptaker, P.S., Mitchell, J. and Mulheron, M. (2007) A unilateral NMR magnet for sub-structure analysis in the built environment: the surface GARField. Journal of Magnetic Resonance, 185, 1-11.

11 Marble, A.E., Mastikhin, I.V., Colpitts, B.G. and Balcom, B.J. (2007) A compact permanent magnet array with a remote homogeneous field. Journal of Magnetic Resonance, 186, 100-4. 
12 Manz, B., Coy, A., Dykstra, R., Eccles, C.D., Hunter, M.W., Parkingso, B.J. and Callaghan, P.T. (2006) A mobile onesided NMR sensor with a homogeneous magnetic field: The NMR-MOLE. Journal of Magnetic Resonance, 183, 25-31.

13 Coates, G.C., Xiao, L. and Prammer, M.G. (1999) NMR Logging Principles and Applications, Halliburton Energy Services, Houston.

14 Appelt, S., Kühn, H., Häsing, F.W. and Bluemich, B. (2006) Chemical analysis by ultrahigh-resolution nuclear magnetic resonance in the Earth's magnetic field. Nature Physics, 2, 105-9.

15 Shushakov, O.A. (1996) Groundwater NMR in conductive water, Geophysics, 61, 998-1006.

16 Callaghan, P.T., Eccles, C.D. and Seymour, J.D. (1997) An Earth's field NMR apparatus suitable for pulsed gradient spin echo measurements of self-diffusion under Antarctic conditions. Review of Scientific Instruments, 68, 4263-70. 
